\title{
Energy Efficient CM2M Communications in E-healthcare Systems
}

\author{
S. Alabadi, R. Wu and Y. Habtay \\ Faculty of Engineering and Science \\ University of Greenwich, Chatham, \\ Kent ME4 4TB, U.K. \\ Email: [Sajad.Alabadi, R.Wu]@greenwich.ac.uk
}

\begin{abstract}
In this paper, cognitive machine to machine (CM2M) gateways in e-healthcare systems are presented by accessing a number of channels via periodic sensing and spectrum handoff. An optimal energy efficient spectrum management mechanism with multiple thresholds is proposed. The developed mechanism aims to reduce energy consumption in the system by optimizing the spectrum sensing and the channel switching, while decreasing the probability of collision, with assuring the reliability thresholds, throughput, and delay. Subsequently an antenna selection sensing (ASS) scheme is used to improve sensing accuracy. The simulation results show that the energy efficiency of the CM2M gateways can be improved significantly.
\end{abstract}

Keywords - M2M; Cognitive Radio; CM2M; Energy efficiency; e-healthcare application.

\section{INTRODUCTION}

The number of machines connected to the global network has been increased massively in the last few years. In 2012 there were 8.7 billion machines connected to the internet, and due to the growth of tablet PCs, smart devices, sensors, the number of machines reached 28.4 billion in 2017 [1]. M2M applications in medical sector become more significant for private and public entities, such as blood pressure and blood oxygen levels, temperature, and electrocardiograms (ECG) [2]. This leads to better efficiency in the e-healthcare systems like early patient discharge from hospitals and faster returns to their normal lives, reducing the total cost of the patient's family and the government spending.

However, the use of wireless M2M communications in ehealthcare systems creates new and complex interference scenarios. This intricacy is generated by medical devices which are normally sensitive to electromagnetic interference (EMI) [3] caused by wireless antennas. The interference can cause many problems to the medical machines (e.g. waveform distortion, howling automatic shutdown, and automatic restart), which can be hazardous to patients using healthcare services. Furthermore M2M applications in healthcare systems, need to be energy efficient and able to work for a long period of time (e.g. 10 years) without battery replacement, while at the same time the Quality of Service (QoS) should be sufficient to ensure the reliability thresholds required in e-healthcare applications. Cognitive radio smart technology can help to address all the aforementioned challenges. E-healthcare applications can benefit from the new functionality that can be achieved by the combination of cognitive radio and $\mathrm{M} 2 \mathrm{M}$ communications (CM2M), CM2M can help to avoid spectrum interference and improve spectrum and energy efficiency in the e-healthcare system [11].

A number of studies have proven that a "spectrum hands-off" operation can increase the throughput of the secondary users (e.g. CM2M gateways) when a number of channels available for spectrum sharing [4], where the CM2M gateways can switch to another free channel and proceed data transmission in case the current used channel busy.

The use of CM2M in e-healthcare applications has recently gained attention in published literature [5]-[7]. In [5] Weightless technology is addressed, considering e-healthcare applications in CM2M communication over TVWS bands for improved spectrum utilisation and longer battery life. In [6] CR and M2M technologies are discussed, including the features they can offer to the medical sector. In [7] mechanisms are suggested for improving energy efficiency in CM2M applications; the mechanisms mainly focus on spectrum discovery schemes such as non-cooperative, cooperative and time-division energy-efficient schemes. To the best of the authors' knowledge, small consideration has as yet been given to the subject of energy efficiency in CM2M e-healthcare applications, especially in relation to using spectrum handoffs and the wait/switch tradeoff with multiple CM2M gateways. In addition, no previous work has considered the scenario with multiple CM2M gateways or addressed delay and the collision among them.

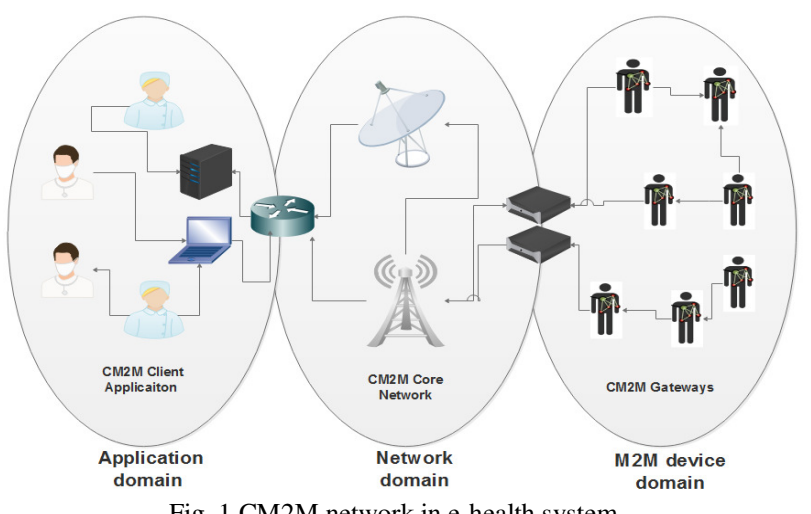

Fig. 1 CM2M network in e-health system 
In this paper, we propose an energy efficient spectrum management mechanism for CM2M e-healthcare system (Fig. 1) by optimising spectrum sensing and switching mechanisms. The proposed mechanism guarantees sensing reliability, throughput, delay and collision probability thresholds simultaneously. The rest of the paper is organised as follows: Section II describes the system model and explains spectrum sensing and access mechanisms. Section III formulates the optimisation problem, while section IV proposes a solution. Discussions and simulation results are illustrate in Section V, and Section VI concludes the paper.

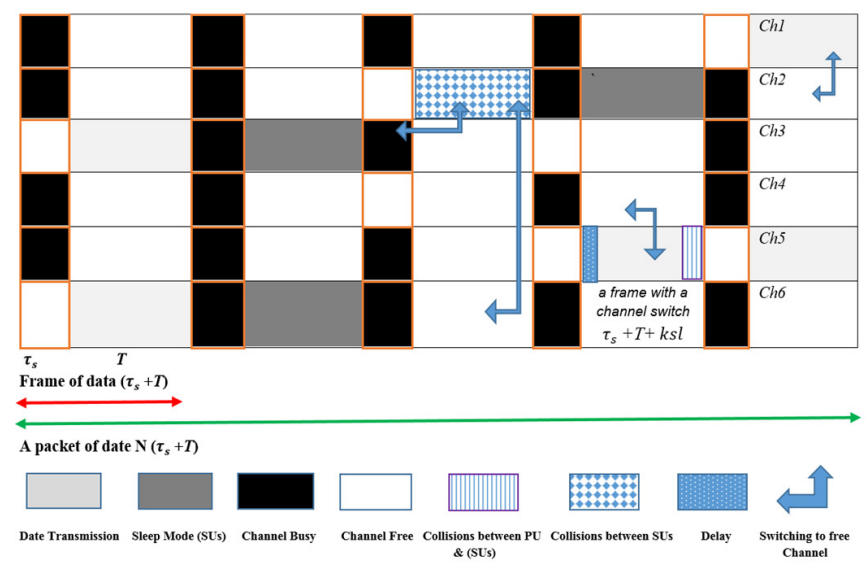

Fig. 2 CM2M gateways performance in transmitting one packet of data

\section{SYSTEM MODEL}

We suppose a CM2M system working in an e-healthcare environment with two CM2M gateways and one primary link. Consider that there are $\mathrm{M}$ channels shared between $\mathrm{CM} 2 \mathrm{M}$ gateways and a primary user. Energy detection assumed to sense the channels, while the CM2M gateways apply wideband sensing to obtain the availability status of all channels. The additive noise is a zero-mean CSCG process. We are interested in a low SNR regime (y) and we assume that the secondary transmission is slotted in via periodic sensing at a specific time, each frame consisting of a sensing slot of durations $\tau_{s}$ and a transmission slot of duration $\mathrm{T}$, where one of the $\mathrm{M}$ channels is allocated to the CM2M gateways.

We assume the spectrum handoff delay $k s l$, is also added after the sensing slot in the CM2M gateways frames if the CM2M gateways decide to carry out channel switching after considering the sensing result and other thresholds. The CM2M gateways at the beginning of each transmission slot may choose to transmit data on the current channel, switch to another channel with a switch delay of $k s l$, or stay on the current channel without transmitting; the primary transmission follows an on-off traffic model and is assumed to be continuous [8]. Fig. 2 shows the CM2M gateways performance in transmitting one packet of data.

We assume the average received signal-to-noise ratio (SNR) of the PU's signal for all channels, the false alarm probability $P f$ and the detection probability $P d$ are the same during one packet of data transmission [8]. The received signal presume sampled at sampling frequency $f s$, the frequency band with carrier frequency fc and bandwidth $\mathrm{Wk}$.
The goal is to decrease energy consumption of the CM2M gateways to transmit a packet of data under the probability of both detection and false alarm thresholds, as discussed in [9], proposing that we can jointly optimize the sensing slot length $\tau_{s}$ and the stay probability on the current channel $L_{s}$ so that the energy consumption of one complete data packet transmission is reduced as follows:

$$
\left(\tau_{s}^{\text {optimal }}, L_{s}^{\text {optimal }}\right)=\min _{\tau_{s}, L_{s}} X\left(\tau_{s}, L_{s}\right)
$$

where $L_{s}{ }^{\text {optimal }}$ and $\tau_{s}{ }^{\text {optimal }}$ are the optimal stay probability and sensing slot length, respectively, while $\mathrm{X}$ is CM2M gateways total average energy cost of one data packet transmission, which includes spectrum sensing, spectrum handoff, and data transmission. It is worth noting that by applying energy detection, the energy consumption caused by the sensing process can be calculated by the length of the sensing slot. By assigning a target false alarm probability $P f t$ and a target detection probability $P d t$ (e.g., $P f t=$ 0.1 and $P d t=0.9$ ) for each channel, we can consider the time required to satisfy the sensing accuracy requirements while reducing this energy cost [9] as given below:

$$
\tau_{s}^{\min }=\frac{1}{y f_{s}}\left(Q^{-1}\left(\bar{P}_{f}\right)-Q^{-1}\left(\bar{P}_{d}\right) \sqrt{2 y+1}\right)
$$

\section{PROBLEM}

By taking into consideration the challenges and the design features in the aforementioned sections, we now present the proposed energy efficient mechanism to manage the available spectrum. We first expand the energy consumption $X$ of the CM2M gateways, given in (1), as follows:

$$
X\left(\tau_{s}, L_{s}\right)=V E_{t}+\mathrm{N} \tau_{s} E_{s}+N P_{x} J_{s w}
$$

where $E_{t}$ and $E_{s}$ are the power (energy cost per second in Watts) due to data transmission and sensing respectively, and $J_{s w}$ is the energy cost for one channel switching, in joules [8,9]. Assuming that $E_{t}, E_{s}$ and $J_{s w}$ are known for a given CM2M ehealthcare system, $N$ is the number of frames and the number of transmission slots of duration $T$ seconds required to complete one packet transmission in the presence of PU. $V$ is the time duration (in the unit of seconds) of one data packet transmission without the PU's presence, and $P_{x}$ is the probability that the CM2M gateways choose to switch to a sensed-as-idle channel when the current channel is sensed as busy and at least one of the other channels is sensed as idle. As shown in (1), the CM2M gateways minimal energy cost can be obtained by jointly optimizing $\tau_{s}$ and $L_{s}$ under reliability thresholds. Despite this it is not a convex optimization problem under all thresholds, for a given $\tau_{s}$, the lower energy consumption can always be found by employing the optimal $L_{S}$ as $L_{S}{ }^{\text {optimal }}=L_{S}{ }^{\text {upper }}$.

And the reason for that function $\mathrm{X}$ become smaller as a function of $L_{s}$. Thus, the minimum value of $\mathrm{X}$ happens at the farthest possible value of $L_{s}$. Because $L_{S}{ }^{\text {optimal }}=L_{S}{ }^{\text {upper }}$ this reveals that the probability of collision threshold is inactive in deriving $L_{s}{ }^{\text {upper }}$ [11]. Furthermore, the probability of collision threshold is vital to get the shortest sensing slot, where $L_{s}$ minimum $\left(\tau_{s}\right)$ is calculated from the probability of collision threshold. As a 
conclusion the shortest $\tau_{\mathrm{s}}$ required to meet all reliability thresholds is given by:

$$
\tau_{s}^{\min , 2}=\arg _{\tau_{s}} \min \left\{L_{s}^{\text {lower }}\left(\tau_{s}\right) \leq L_{s}^{\text {upper }}\left(\tau_{s}\right)\right\}
$$

where $\mathrm{L}_{\mathrm{s}}$ minimum $\left(\tau_{\mathrm{s}}\right)$ is calculated by the collision threshold as a conclusion the minimum $\tau_{\mathrm{s}}$ meet collision threshold, detection probability Pdt and false alarm probability Pft is given by:

$$
\tau_{s}^{\min }=\max \left\{\tau_{s}^{\min , 1}, \tau_{s}^{\min , 2}\right\}
$$

Note that $\tau_{s}^{\mathrm{min}, 1}$ is calculated by the reliability thresholds: the probability of detection and the false alarm probability from (2). We can get $\tau_{s}^{\min , 2}$ from the other reliability thresholds defined as the target throughput, probability of collisions, and delay.

\section{SOLUTION}

Considering all the aforementioned thresholds, it will require longer $\tau_{s}$, to satisfy the conditions of $\left(L_{s}^{\text {lower }} \leq L_{s}\right.$ upper $)$ ensuring a feasibility region of $L_{s}, \Delta\left(\tau_{s}\right)=\left(L_{s}{ }^{\text {upper }}\left(\tau_{s}\right) \leq\right.$ $L_{s}{ }^{\text {lower }}\left(\tau_{s}\right) \geq 0$ ) and perhaps for practical values of $\tau_{s}$ no solution can optimize the existing problem. Furthermore, it is justified by the fact that, for a given sensing slot duration and false alarm probability, the probability of collision diminishes monotonically with any rise in the probability of detection value. Therefore, any increase in detection probability leads to a lower $L_{s}{ }^{\text {lower }}$. Moreover, by enhancing the probability of detection, feasibility region $\Delta\left(\tau_{s}\right)$ becomes obtainable with shorter sensing slot time, which as results decreases the energy consumption caused to by the sensing process.

There are many schemes to improve the probability of detection other than a typical single antenna sensing, like multi-antenna parallel sensing and cooperative sensing, but as we work under CM2M concepts and goals we must avoid extra signalling to decrease cost and hardware complexity when run a number of RF chains. Ideal possible solution here comes by applying an ASS scheme to relief the bounds of $L_{s}$. The ASS use J antennas and $\mathrm{Z} \mathrm{RF}$ chains $(\mathrm{Z}<J)$ for the sensing process, where the CM2M gateways senses the channels for duration of time $\left(\tau_{s}\right)$. Furthermore, the sensing slot of $\tau_{s}$ period is divided into $\mathrm{J} / \mathrm{Z}$ sub-slots of period $\tau_{a s}$ (i.e., $\tau_{s}=J / Z \tau_{a s}$ ).

$\mathrm{Z}$ antennas are used to proceed spectrum sensing in each subslot at the same time. For instance, we suppose $Z=1$ and $J=2$. Comparing with the multi-antenna (conventional) spectrum sensing (i.e., $J$ antennas are employed for sensing at the same time employed for $\mathrm{W}=K \mathrm{RF}$ chains), the developed mechanism can decrease hardware complexity and cost, since only one or a subset of RF chains are employed with huge number of antennas $[10,11]$.

\section{SIMULATION}

In this section, we show the efficiency of our mechanism using Matlab simulations. The numerical results are given here to prove the analysis in the previous sections. The primary user is assumed to be a QPSK modulated signal with a bandwidth of $7 \mathrm{MHz}$. The sampling frequency is the same as the bandwidth of the primary user. We consider the power required for spectrum sensing and transmission are $E_{t}=65 \mathrm{~mW}$ and $E_{s}=45 \mathrm{~mW}$ respectively.

TABLE. 1 VALUE SETTING OF SIMULATION

\begin{tabular}{|c|c|c|}
\hline Parameter & Value & Description \\
\hline $\mathrm{M}$ & 6 & Available channel number \\
\hline $\mathrm{v}$ & $6 \mathrm{~s}$ & Duration of one packet data \\
\hline $\mathrm{T}$ & $0.8 \mathrm{~s}$ & Duration of each transmission \\
\hline$\gamma$ & $-10 \mathrm{~dB}$ & SNR \\
\hline $\mathrm{J}_{\mathrm{SW}}$ & $40 \mathrm{~mJ}$ & The energy cost for one channel switching \\
\hline$\rho$ & 0.4 & The probability of a channel being busy \\
\hline $\mathrm{P}_{\mathrm{d}}$ & 0.9 & The target probability of detection \\
\hline $\mathrm{P}_{\mathrm{f}}$ & 0.1 & The target probability of false alarm \\
\hline
\end{tabular}

Fig. 3 shows ASS $(J=2)$ can increase the detection probability which ultimately leads to a smaller probability of collision than Single Antenna Sensing SAS $(J=1)$ with the same value of $\tau_{s}$.

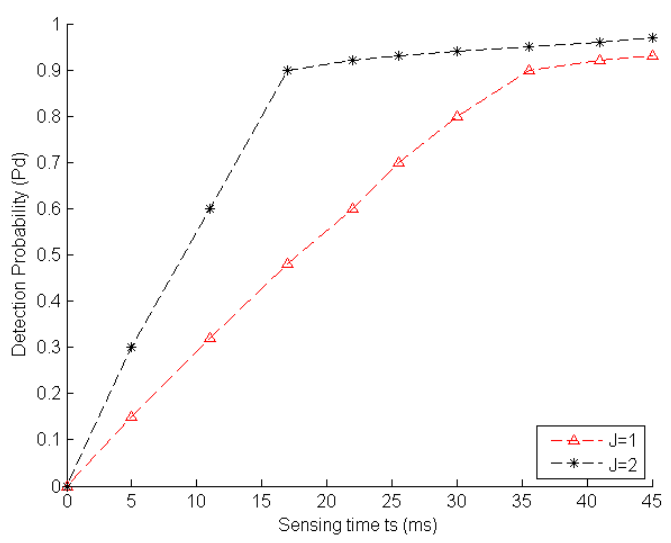

Fig. 3 Using Antenna- selection sensing $(J=2)$

This easily can be explained by the relationship between the probability of collision and the probability of detection as the probability of collision value decrease with any increase in probability of detection, and shows that ASS needs lower $\tau_{s}$ and energy consumption than the traditional antenna (single) sensing when achieving similar probability of collision thresholds.

Moreover, for a given $L_{s}$, the probability of collision also monotonically getting smaller with $\tau_{s}$. In fact, this easily can be explained because a higher value of $\tau_{s}$ leads to a higher probability of detection value and from above we know that will ultimately lead to decreases the probability of collision. Bear in mind probability of collision mainly caused from CM2M gateways switching to a channels with primary user (PU), due to failed detection.

Fig. 4 plots the $L_{s}$ bounds of the CM2M gateways as function of the sensing time $\tau_{s}$ for SAS $(J=1)$, and ASS $(J=2)$. Using the same $\tau_{s}, L_{s}$ lower increased when additional stringent probability of collision thresholds $\mathrm{Q}$ (i.e, from $\mathrm{Q}=0.4$ to $\mathrm{Qz}$ $=0.3$ under $J=2$ in Fig. 4) used.

Such an increase in $L_{s}^{\text {lower }}$, because of the additional stringent $\mathrm{Q}$, allows the CM2M gateways to remain on the current channel for a longer time to keep the probability of collision thresholds. The upper bound $L_{s}^{\text {upper }}$ is decided by measuring which 
thresholds (delay or throughput) is more significant at given time. Fig. 4 consider the throughput $\left(\tau_{s}, L_{s}\right.$ upper $)$ thresholds.

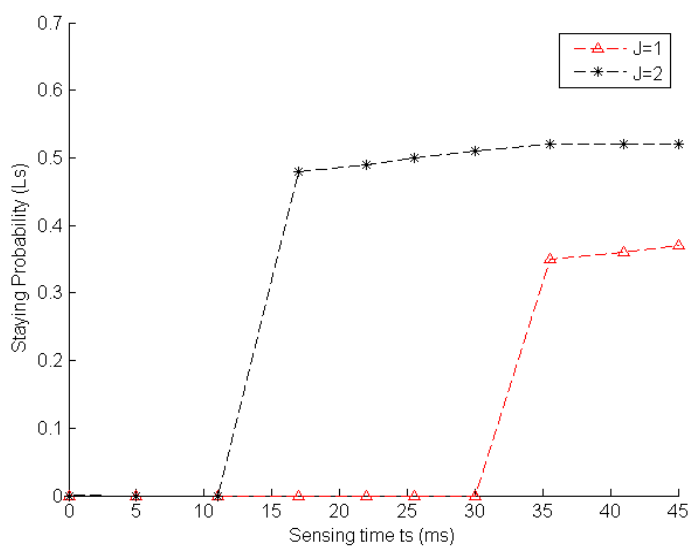

Fig. $4 L_{s}$ Bounds of the CM2M gateways as a function $\tau_{s}$

Bearing in mind that any delay will yield a decrease in average throughput, and therefore the CM2M gateway will need to switch its channel repeatedly to meet its required throughput thresholds. The feasible region becomes available at $\tau_{s}=16$ $(\mathrm{ms})$ if $\operatorname{ASS}(J=2)$ is used. While using (SAS) $(J=1)$, the feasibility value can only achieve at $\tau_{s}=41 \mathrm{~ms}$ ). This confirms what we mentioned in the previous section stating that, by using the ASS, the bounds of $L_{S}$ can be relief. Alike, the energy consumption in CM2M gateways due to sensing can be considerably reduced, as shown in Fig. 5.

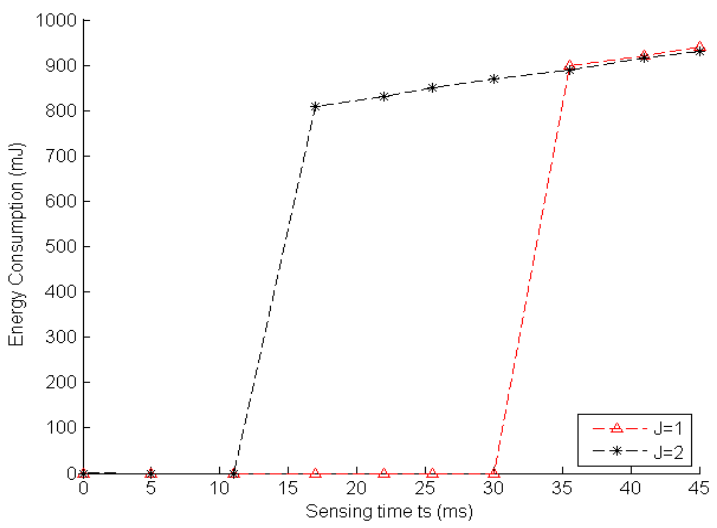

Fig. 5 The energy consumption of the CM2M gateways under various sensing mechanisms

Fig. 5 shows the energy consumption of the CM2M gateways spectrum usage under various sensing mechanisms. It proves that the best stay probability $\tau_{s}$ can be found at $L_{s}$ upper. As explained before, the optimal sensing slot $\tau_{s}^{\text {optimal }}$ is also calculated by the reliability thresholds. As seen in Fig. 5 the ASS offers reduced sensing time $\left(\tau_{s}=16 \mathrm{~ms}\right)$ to meet all the thresholds, compared to SAS $\left(\tau_{s}=41 \mathrm{~ms}\right)$. Greater $L_{s}=0.48$ can be satisfied while meeting all the thresholds with smaller $\tau_{s}$ by using ASS.

As a total outcome, the energy efficiency of the CM2M gateways can be improved significantly by using ASS under the same thresholds.

\section{CONCLUSION}

In this paper an energy efficient spectrum management mechanism for CM2M e-healthcare system has been designed. The designed mechanism simultaneously considers the wait/switch trade-off in terms of channel switching probability, sensing /throughput trade-off in terms of the period of sensing time and collision probability.

Furthermore, the proposed mechanism considers an ASS scheme for improved sensing accuracy and reduced energy consumption. The simulation results have shown the improved optimality and efficiency of our mechanism under the desired thresholds.

\section{REFERENCE}

[1] J. Wang, M. Ghosh and K. Challapali, "Emerging cognitive radio applications: A survey", in IEEE Communications Magazine, Vol. 49, No. 3, pp. 74-81, March 2011

[2] J. Palicot and C. Roland.( 2005, October 5), "On the use of cognitive Radio for Decreasing Electromagneti- Radiation".[online]: Available: https://hal.archives-ouvertes.fr/hal-00776220.

[3] H. Furahata, "Electromagnetic interferences of electric medical equipment from hand-held radiocommunication equipment", 1999 International Symposium on Electromagnetic Compatibility (IEEE Cat. No.99EX147), Tokyo, 1999, pp. 468-471..

[4] M. Guerrini, L. Rugini, and P. Banelli, "Sensing-throughput tradeoff for cognitive radio networks", IEEE SPAWC, Darmstadt, Germany, June 2013.

[5] I. Poole, "Weightless Wireless M2M White Space Communications" (May 2015), [Online]: Available: http://www.radio-electronics.com/info/ wireless/weightless.

[6] J. Palicot and C. Roland, (July 2014), "On the Use of Cognitive Radio for Decreasing Electromagnetic-Radiation”, [Online]: Available http:// www.ursi.org/Proceedings/ProcGA05/pdf/C05.5(0534).

[7] R. Lu, X. Li, X. Liang, X. Shen and X. Lin, "GRS: The green, reliability, and security of emerging machine to machine communications", IEEE Commun. Mag., Vol. 49, No. 4, pp. 28-35, Apr. 2011.

[8] W. D. de Mattos, P. R. L. Gondim, "M-Health solutions using 5G networks and M2M communications", pp. 24-29, DOI: 10.1109/MITP.2016.52 2016.

[9] S. Alabadi, P. Rapajic, K. Arshad and S. Rostami, "Energy efficient cognitive M2M communications", International Journal of Interdisciplinary Telecommunications and Networking (IJITN), Vol. 8, No. 3, pp. 1-9, July 2016.

[10] S. Wang et al., "Antenna selection based spectrum sensing for cognitive radio networks", 22th IEEE International Symposium on Personal, Indoor and Mobile Radio Communications, Sept. 2011, pp. 364-68.

[11] S. Wang, F. Tosato and J. P. Coon, "Reliable energy-efficient spectrum management and optimization in cognitive radio networks: How often should we switch", IEEE Wireless Communications, Vol. 20, No. 6, pp. 14-20, 2013. 